\title{
Predicting e-Mail Response Time in Corporate Customer Support
}

\author{
Anton Borg ${ }^{1} \mathbb{1}^{\mathrm{a}}, \mathrm{Jim}$ Ahlstrand ${ }^{2}$ and Martin Boldt ${ }^{1} \mathbb{1}^{\mathrm{b}}$ \\ ${ }^{1}$ Blekinge Institute of Technology, 37179 Karlskrona, Sweden \\ ${ }^{2}$ Telenor AB, Karlskrona, Sweden
}

Keywords: e-Mail Time-to-Respond, Prediction, Random Forest, Machine Learning, Decision Support.

Abstract: Maintaining high degree of customer satisfaction is important for any corporation, which involves the customer support process. One important factor in this work is to keep customers' wait time for a reply at levels that are acceptable to them. In this study we investigate to what extent models trained by the Random Forest learning algorithm can be used to predict e-mail time-to-respond time for both customer support agents as well as customers. The data set includes 51,682 customer support e-mails of various topics from a large telecom operator. The results indicate that it is possible to predict the time-to-respond for both customer support agents (AUC of 0.90) as well as for customers (AUC of 0.85). These results indicate that the approach can be used to improve communication efficiency, e.g. by anticipating the staff needs in customer support, but also indicating when a response is expected to take a longer time than usual.

\section{INTRODUCTION}

An important element in any corporation is to maintain high-quality and cost-efficient interaction with the customers. This is especially important for interactions between the organization and customer via customer support, since failing to resolve customers issues satisfactorily risk negatively affecting the customers view of the organization. Further, in a prolongation this might affect the overall reputation of the organization. In highly competitive markets, a single negative customer service experience can deter potential new customers from a company or increase the risk of existing customers to drop out (Halpin, 2016), both negatively affecting the volume of business.

For many customers e-mails still account for an important means of communication due to both its ease and widespread use within almost all age groups (Kooti et al., 2015). As such, implementing efficient customer service processes that target customer e-mail communication is a necessity for corporations as they receive large numbers of such customer service e-mails each day. Furthermore, the customers expect short response times to digital messages, which further complicates the customer service process (Church and de Oliveira, 2013).

a(D) https://orcid.org/0000-0002-8929-7220
b(iD) https://orcid.org/0000-0002-9316-4842
In this study we investigate the possibility to use supervised machine learning in order to predict when an e-mail response will be received, time-to-respond (TTR) or responsiveness. The semi-automated customer service e-mail management system studied exists within one of the bigger telecom operators in Europe with over 200 million customers worldwide, and some 2.5 million in Sweden. When these customers experience problems they often turn to e-mail as their means of communication with the company, by submitting an e-mail to a generic customer service e-mail address. Under-staffing might impact the efficiency of customer support, negatively impacting customer relations. However, while over-staffing might produce quick responses, it might also result in customer support agents being idle. Consequently, it is important to be able to predict the customer support workload in order to successfully schedule personnel and improve communication efficiency (Yang et al., 2017).

Customer service e-mails, provided by the telecom company, contains support errands with different topics. Each customer service e-mail might contain different topics, and the importance of each topic might be of varying importance, depending on the customer. Different topics require different actions by customers, and thus would require varying time before a response can be expected. The content of an e-mail within a topic, e.g. invoice, might also affect time-to-respond, as certain actions are more compli- 
cated than others. Further, a customer service e-mail might contain two paragraphs of text, one detailing a technical issue, and the other one an order errand. As such, the e-mail topic would be sorted as Invoice, Techicallssue, and Order. This would further affect response time.

\subsection{Aims and Objectives}

In this study we investigate the possibility to predict the time-to-respond for received e-mails based on its content. If successful, it would be possible to adjust the schedules for customer support personnel in order to improve efficiency. The two main questions investigated in this work are as follows. First, to what extent it is possible to predict the time required by customer support agents to respond to e-mails. Second, to what extent it is possible to predict the time it takes customers to respond to e-mails from customer support personnel.

\subsection{Scope and Limitations}

The scope of this study is within a Swedish setting, involving e-mail messages written in Swedish sent to the customer service branch of the studied telecom company. However, the problem studied is general enough to be of interest for other organizations as well. In this study, e-mails where no reply exists have been excluded, as it has been suggested to be a separate classification task (Huang and $\mathrm{Ku}, 2018$ ). Further, time-to-respond (TTR) is investigated independent of the workload of agents, and the content of the e-mails.

\section{RELATED WORK}

Time-to-Respond, or responsiveness, can affect the perceived relationship between people both positively and negatively (Church and de Oliveira, 2013), (Avrahami and Hudson, 2006), (Avrahami et al., 2008).

Investigations into mobile instant messaging (e.g. SMS) indicates that it is possible to predict whether a user will read a message within a few minutes of receiving it (70.6\% accuracy) (Pielot et al., 2014). This can be predicted based on only seven features, e.g. screen activity, or ringer mode.

Responsiveness to IM has been investigated, and been predicted successfully ( $90 \%$ accuracy) (Avrahami and Hudson, 2006). The paper where limited to messages initiating new sessions, but the model where capable of predicting whether an initiated session would get a response within $30 s, 1,2,5$, or 10 minutes. Predicting the response time when interacting with chatbots using IM have also been investigated, within four time intervals $<10 s, 10-30 s$, $30-300 s$, and $>300 s$ (Accuracy of 0.89), but also whether a message will receive a response (Huang and $\mathrm{Ku}, 2018$ ).

Similarly to IM, response time in chat-rooms have also been investigated, with one study finding that the cognitive and emotional load affect response time within and between customer support agents (Rafaeli et al., 2019). In a customer support setting, the cognitive load denotes e.g. the number of words or amount of information that must be processed. TTR predictions have also been investigated in chat rooms (AUC 0.971 ), intending to detect short or long response times (Ikoro et al., 2017).

However, it seems that there is little research that have investigated predicting the TTR of e-mails in a customer support setting. This presents a research gap as it has been argued that e-mails are a distinct type of text compared to types of text (Baron, 1998). Research indicates that it is possible to estimate the time for an e-mail response to arrive, within the time intervals of $<25 \mathrm{~min}, 25-245 \mathrm{~min}$, or $>245 \mathrm{~min}$ (Yang et al., 2017). Similarly, research has been conducted on personal e-mail (i.e. non-corporate) (Kooti et al., 2015). However, this investigates quite small TTRs which, although suitable for employee e-mails, might not conform to the customer support setting according to domain experts. Further, the workload estimation of customer support agents work resolution benefits from an increased resolution, i.e. more bins.

\section{DATA}

The data set consists of 51,682 e-mails from the customer service department from a Swedish branch of a major telecom corporation. Each e-mail consists of the:

- subject line,

- send-to address,

- sent time, and

- e-mail body text content.

Each e-mail is also labeled with at least one label. In total there exists 36 distinct topic labels, each independent from the others, where several of these might be present in any given e-mail. The topics have been set by a rule-based system that was manually developed, configured and fine-tuned over several years by domain expertise within the corporation. 
Table 1: Description of the Features Extracted or Calculated from the Data Set.

\begin{tabular}{llcl}
\hline Feature name & Type & Value range & Description \\
\hline Text sentiment & Float & {$[-1,+1]$} & Text sentiment of an e-mail ranging negative to positive. \\
Customer escalated & Boolean & $\{0,1\}$ & Whether customer changed between messages in thread. \\
Agent escalated & Boolean & $\{0,1\}$ & Whether agent changed between messages in thread. \\
Old & Boolean & $\{0,1\}$ & Whether a message is older than 48 hours, or not. \\
Text complexity & Float & {$[0,100]$} & Indication of the text complexity. \\
Sender & Categorical & Text & The e-mail address of the sender. \\
Message length & Integer & $\geq 1$ & Number of characters in each e-mail message. \\
\hline
\end{tabular}

A DoNotUnderstand topic label acts as the last resort for any e-mail that the current labeling system is unable to classify. Those e-mails have been excluded from the data set and each e-mail has been anonymized. Further, ends of threads have been excluded from the data set (i.e. e-mails where no reply exists), as that has been suggested to be a separate classification task (Huang and $\mathrm{Ku}, 2018$ ).

The e-mails are grouped into conversation threads, and for each e-mail the date and time sent is available, enabling the construction of a timeline for each thread. Further, it is possible to shift the time-date information in each thread by one step, so that the future sent time is available for each e-mail in the thread. As such, this data can be considered the TTR. In order to adjust the resolution of the TTR, the datetime where binned into groups (Avrahami and Hudson, 2006). The bins were decided by consulting with the telecom company and thus using their domain knowledge. Six bins where utilized: response within 2 hours, between $2-4$ hours, between $4-8$ hours, between $8-24$ hours, between $24-48$ hours, and more than 48 hours. The bins are considered as the class labels.

The data set is divided into subsets, by topic and sender. The topics Credit $(n=6,239), \operatorname{Order}(n=$ $2,221)$, and ChangeUser $(n=1,398)$ are used to investigate this problem. Further, similar to the work by Yang et al. each topic is divided into one set for e-mails sent from the telecom corporation and another set for e-mails sent by the customer (Yang et al., 2017). In this case, the agents can be considered a more homogeneous group (similar training and experience), whereas the customers could be regarded as a heterogeneous group (different background and experiences). As such, six data sets have been created.

The class distribution in the data set is exemplified by ChangeUser topic in Figure 1 for agents and Figure 2 for customers. A majority of the messages have a TTR within two hours, followed by a TTR longer than 48 hours, $8-24$ hours. A minority of messages have a TTR between $2-4,4-8$, or $24-48$ hours. Consequently, it would seem that messages get responses "immediately", the next day, or after two days.

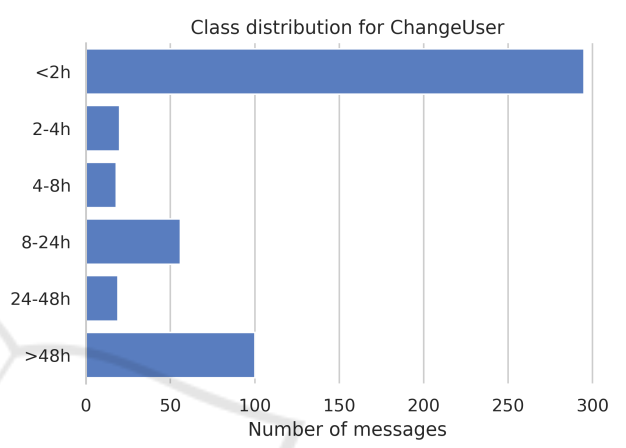

Figure 1: ChangeUser Agent TTR Class Distribution.

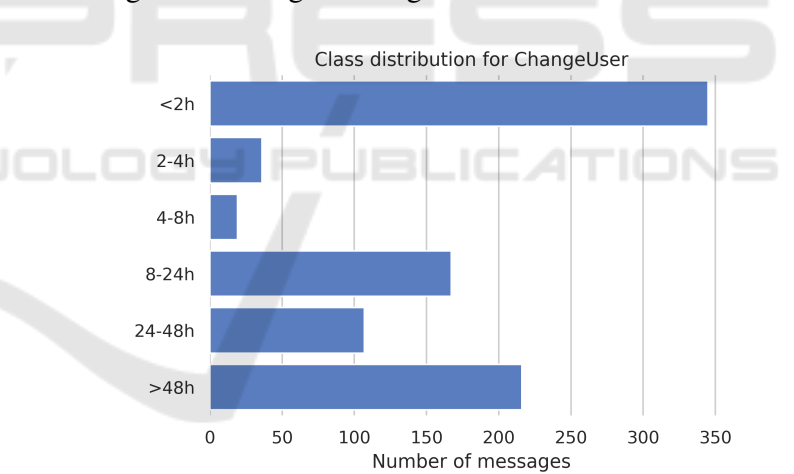

Figure 2: ChangeUser Customer TTR Class Distribution.

\subsection{Feature Extraction}

For each e-mail in the data set, seven features are calculated or extracted. A summary of all features used in the study are shown in Table 1. First, Vader sentiment is used to calculate the Text sentiment for each email (Hutto and Gilbert, 2015), (Rafaeli et al., 2019). As the primary language in this data set is Swedish, a list of Swedish stop-words was used ${ }^{1}$. However, the Swedish stop-words were extended by English stopwords, as a fair amount of English also occurs due to

\footnotetext{
${ }^{1}$ https://gist.github.com/peterdalle/8865eb918a824a475 b7ac5561f2f88e9
} 
the corporate environment.

Second, for each message it was calculated whether the customer or support agent responding participating in the conversation had changed over the thread timeline, denoted by the Boolean variables Customer escalated and Agent escalated respectively. A change in e.g. customer support agent indicates the involvement of an agent experienced in the current support errand. However, related work indicates that as the number of participants increase, so do the time to respond (Yang et al., 2017). The variable Old denotes if the message has not received a response for 48 hours or more, as per internal rules at the company. A Text complexity factor for the text is also calculated as per

$$
C F=\frac{|\{x\}|}{|x|} \times 100,
$$

where $x$ is the e-mail content (Abdallah et al., 2013). Consequently, Equation 1 is the number of unique words in the e-mail divided by the number of words in the e-mail. A higher score indicates a higher complexity in the text, which can affect the TTR (Rafaeli et al., 2019). It should be noted that there exist different readability scores for the English language, e.g. Flesch-Kincaid score (Farr et al., 1951). However, the applicability of these on Swedish text is unknown. Finally, the Sender and Length of the e-mail is also included as variables.

\section{METHOD}

This section describes the experimental approach, which includes for instance the design and chosen evaluation metrics.

\subsection{Experiment Design}

Two experiments with two different goals were included in this study. The first experiment aimed to investigate whether it is possible to predict the time a customer support agent would take to respond to the e-mail received. As such, the experiment used the data set containing e-mails sent by the customer and tried to predict when the agent would respond. In this experiment the independent variable was the models trained by the learning algorithms described in Section 4.2. The dependent variables were the evaluation metrics described in Section 4.4, of which the AUC metric was chosen as primary.

The second experiment is similar to the first one, but instead uses the data sets containing e-mails sent by the customer support agents, thus aiming to predict when the customer will respond. As such both the independent and the dependent variables were the same as in the first experiment.

Evaluation of the classification performance was handled using a 10-times 10-fold cross-validation approach in order to train and evaluate the models (Flach, 2012). Each model's performance was measured using the metrics presented in Section 4.4.

\subsection{Included Learning Algorithms}

Random Forest (Breiman, 2001) was selected as the learning algorithm to investigate in this study. It is a suitable algorithm as the data contains both Boolean, categorical, and continuous variables. Initially a SVM model (Flach, 2012) was also evaluated, but since Random Forest significantly outperformed the SVM models, they were excluded from the study. The reason to why the SVM model showed inferior performance is not clear. Although it is in line with the "No free lunch" theorem, stating that no single model is best in every situation. Thus, models' performance varies when evaluated over different problems.

The models trained by the Random Forest algorithm were compared against a Random Guesser classifier using a uniform random guesser as baseline (Pedregosa et al., 2011), (Yang et al., 2017).

\subsection{Class-balance}

In order to deal with the class imbalance of the different bins, a multi-class oversampling strategy was used that relied on SMOTE and cleaned by removing instances which are considered Tomek links (Batista et al., 2003; Lemaître et al., 2017). Using only oversampling can lead to over-fitting of the classifiers as majority class examples might overlap the minority class space, and the artificial minority class examples might be sampled too deep into the majority class space (Batista et al., 2003).

\subsection{Evaluation Metrics}

The models predictive performance in the experiments were evaluated using standard evaluation metrics calculated based on the True Positives (TP), False Positives (FP), True Negatives (TN), and False Negatives (FN). The evaluation metrics consists of the $F_{1}$-score (micro average), Accuracy, and Area under ROC-curve (AUC) (micro average).

The theoretical ground for these metrics are explained by Flach (Flach, 2012). The first metric is 


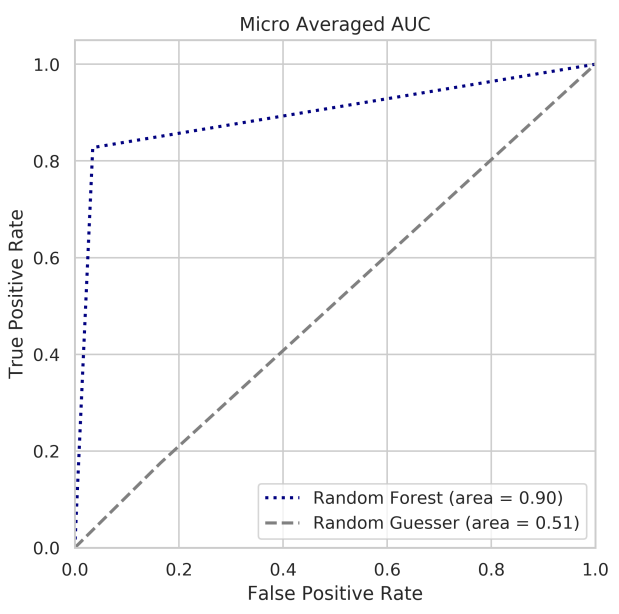

(a) Agent TTR Predictions.

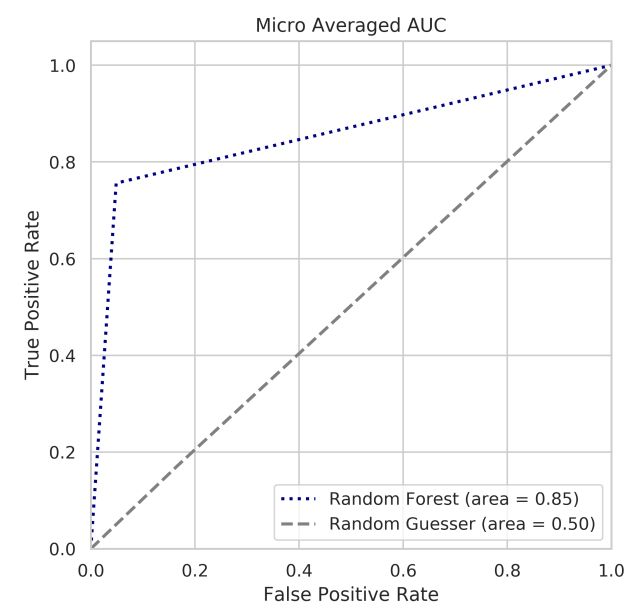

(b) Micro Averaged AUC for Agent Predictions (3a) and Customer Predictions (3) over the Different Topics.

Figure 3: Customer TTR Predictions.

the traditional Accuracy that is defined as in Equation 2 (Yang, 1999):

$$
A c c=\frac{T P+T N}{T P+T N+F P+F N}
$$

It is a measurement of how well the model is capable of predicting TP and TN compared to the total number of instances. For the multi-class case, the accuracy is equivalent to the Jaccard index. Accuracy ranges between $0.0-1.0$, where 1.0 is a perfect score.

However, in cases where there is a high number of negatives, e.g. in a multi-class setting, accuracy is not representative. In these cases the $\mathrm{F}_{1}$-score is often used as an alternative, as it doesn't take true negatives into account (Flach, 2012). Similar to the Accuracy, the $F_{1}$-score ranges between $0.0-1.0$, where 1.0 is a perfect score. It is calculated as described in Equation 5, based on Equation 3 and Equation 4. In this study micro-averaging was used as the number of labels might vary between classes (Yang, 1999).

$$
\begin{gathered}
\text { Precision }=\frac{T P}{T P+F P} \\
\text { Recall }=\frac{T P}{T P+F N} \\
F_{1}=2 * \frac{\text { Precison } * \text { Recall }}{\text { Precision }+ \text { Recall }}
\end{gathered}
$$

For micro-averaging, precision and recall are calculated according to Equation 6 and Equation 7 respectively, where $n$ is the number of classes.

$$
\begin{gathered}
\text { Precision }_{\mu}=\frac{T P_{1}+\ldots+T P_{n}}{T P_{1}+\ldots+T P_{n}+F P_{1}+\ldots+F P_{n}} \\
\text { Recall }_{\mu}=\frac{T P_{1}+\ldots+T P_{n}}{T P_{1}+\ldots+T P_{n}+F N_{1}+\ldots+F N_{n}}
\end{gathered}
$$

Hamming loss measures the fraction of labels that are incorrect compared to the total number of labels (Tsoumakas et al., 2010). A score of 0.0 represents a perfect score as no labels were predicted incorrectly.

The AUC metric calculates the area under a curve, which in this case is the ROC. Hence, the AUC is also known as the Area under ROC curve (AUROC). AUC is often used as a standard performance measure in various data mining applications since it does not depend on an equal class distribution and misclassification cost (Fawcett, 2004). A perfect AUC measure is represented by 1.0 , while a measure of 0.5 is the worst possible score since it equals a random guesser.

\section{RESULTS}

The results are divided into two subsections, one for each of the experiments described in Section 4.1.

\subsection{Experiment 1: Customer Agent Response Prediction}

Figure 3a shows the micro-averaged AUC over the different topics for Random Forest and the random guesser when predicting support agents' e-mail response times. As expected, the random guesser models have a worst-case AUC metric of 0.51. While 


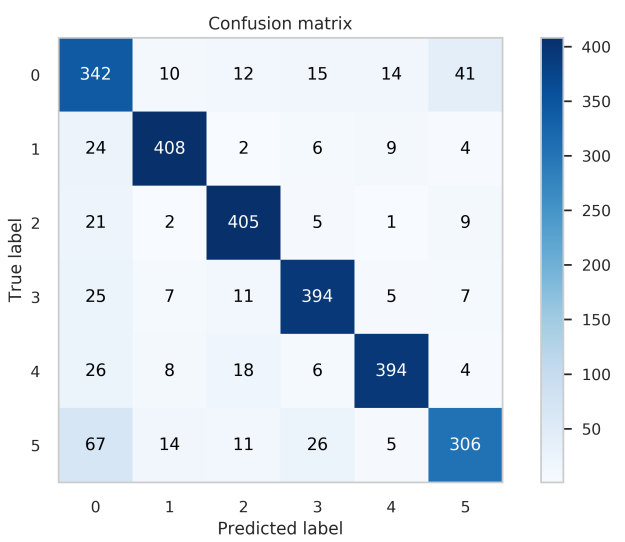

(a) Random Forest.

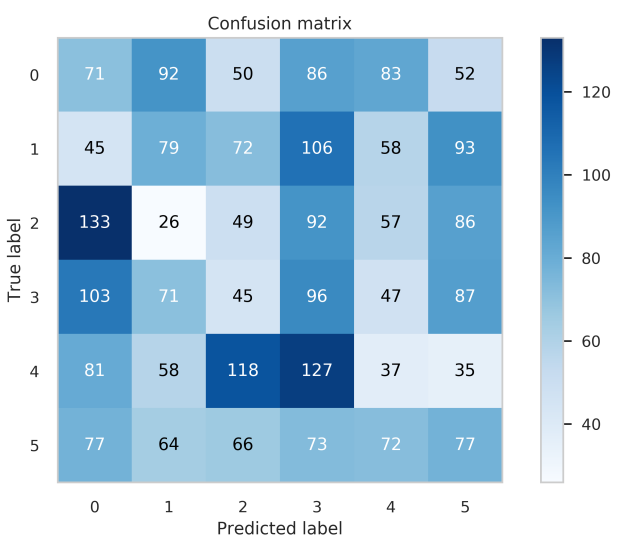

(b) Agent TTR Prediction Performance per Class for Random Forest (4a) and Random Guesser (4a) for the Customer Support Topic Order.

Figure 4: Random Guesser Baseline.

Table 2: Agent Time-to-Reply (TTR) Results.

\begin{tabular}{llrrrr}
\hline Topic & Model & Accuracy $($ std $)$ & AUC $($ std $)$ & F $_{1}$-score $($ std $)$ & Hamming $($ std $)$ \\
\hline \multirow{2}{*}{ ChangeUser } & Random Forest & $0.8720(0.0214)$ & $0.9232(0.0128)$ & $0.8720(0.0214)$ & $0.1279(0.0214)$ \\
& Baseline & $0.1758(0.0324)$ & $0.5055(0.0194)$ & $0.1758(0.0324)$ & $0.8241(0.0324)$ \\
Credit & Random Forest & $0.8149(0.0096)$ & $0.8889(0.0057)$ & $0.8149(0.0096)$ & $0.1850(0.0096)$ \\
& Baseline & $0.1822(0.0082)$ & $0.5093(0.0049)$ & $0.1822(0.0082)$ & $0.8177(0.0082)$ \\
Order & Random Forest & $0.8442(0.0310)$ & $0.9065(0.0186)$ & $0.8442(0.0310)$ & $0.1557(0.0310)$ \\
& Baseline & $0.1535(0.0114)$ & $0.4921(0.0068)$ & $0.1535(0.0114)$ & $0.8464(0.0114)$ \\
\hline
\end{tabular}

the models trained by the Random Forest algorithm show interesting predictive results with an overall AUC metric of 0.90 , which significantly outperforms random chance.

Figure $4 \mathrm{~b}$ shows the absolute confusion matrix for the predicted agent response times vs. the true agent response times for the Random Forest algorithms (Figure 4a) and the Random Guesser baseline (Figure 4). The matrix shows the aggregated results over the different test folds showing that the Random Guesser baseline classifier randomly appoints the classes. In contrast the Random Forest model has a clear diagonal score that indicates significantly better prediction performance compared to the random baseline.

This is supported by the results shown in Table 2 in which the Random Forest models have AUC scores slightly above or below 0.9 . In fact the $95 \%$ confidence interval of the AUC metric for each of the three class labels ChangeUser, Credit and Order were $0.92 \pm 0.026,0.89 \pm 0.011$ and $0.91 \pm 0.037$ respectively. This indicates the the models have a good ability to predict the TTR over various class labels. This is further strengthened when evaluating the predictive performance in terms of accuracy or $F_{1}$-scores instead. Although using these metrics, the Random Forest models still performs well above 0.80 , whereas the Random Guesser baseline models are associated with useless scores at 0.18 , or worse. Figure 4a further indicates that the models have a slightly higher misclassification for label 0 , and 5 compared to the other labels. This indicate that TTR prediction within 2 hours and beyond 48 hours are slightly more difficult to predict.

\subsection{Experiment 2: Customer Response Prediction}

Similar to the previous experiment, Figure 3 shows that the Random Guesser baseline models have an AUC metric of 0.50 while the Random Forest models significantly outperforms that with a metric of 0.85 when predicting customers' e-mail response times. Similar to the results in Section 5.1, Figure 5b shows the absolute confusion matrix for the predicted agent response times vs. the true agent response times for the Random Forest models (Figure 5a) and the Random Guesser baseline models (Figure 5).

The matrix shows the aggregated results over the different test folds that clearly show the increased pre- 


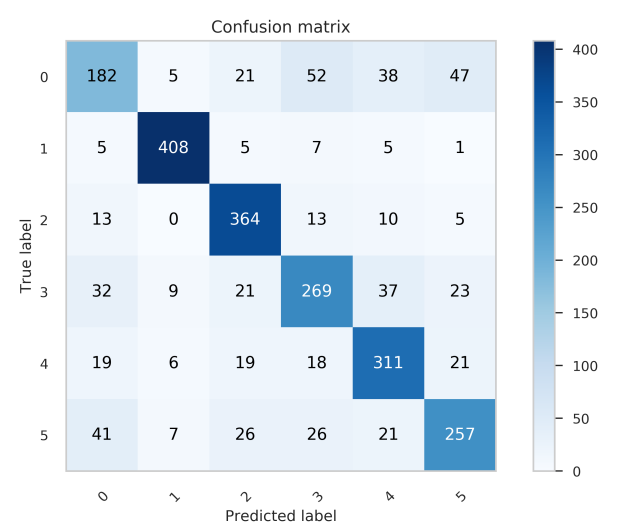

(a) Random Forest.

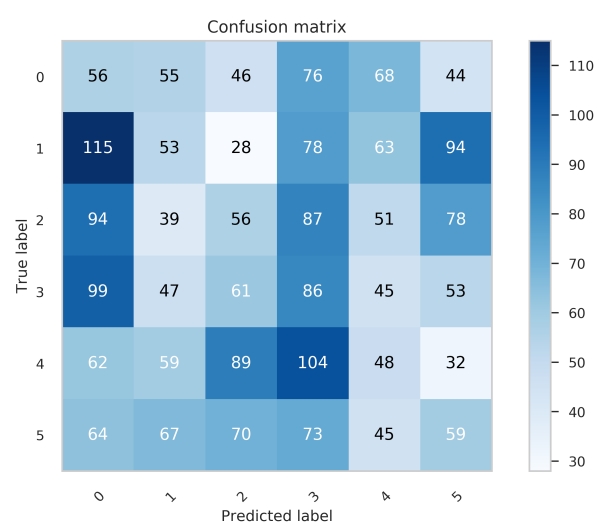

(b) Customer TTR Prediction Performance per Class for Random Forest (5a) and Random Guesser (5) for the Customer Support Topic Order.

Figure 5: Random Guesser Classifier.

Table 3: Customer Time-to-Reply (TTR) Results.

\begin{tabular}{llrrrr}
\hline Topic & Model & Accuracy (std) & AUC $($ std) & F $_{1}$-score $($ std) & Hamming (std) \\
\hline ChangeUser & RF & $0.7760(0.0231)$ & $0.8656(0.0138)$ & $0.7760(0.0231)$ & $0.2239(0.0231)$ \\
& Random & $0.1592(0.0223)$ & $0.4955(0.0134)$ & $0.1592(0.0223)$ & $0.8407(0.0223)$ \\
Credit & RF & $0.7486(0.0088)$ & $0.8491(0.0053)$ & $0.7486(0.0088)$ & $0.2513(0.0088)$ \\
& Random & $0.1798(0.0055)$ & $0.5079(0.0033)$ & $0.1798(0.0055)$ & $0.8201(0.0055)$ \\
Order & RF & $0.7640(0.0154)$ & $0.8584(0.0092)$ & $0.7640(0.0154)$ & $0.2359(0.0154)$ \\
& Random & $0.1527(0.0216)$ & $0.4916(0.0130)$ & $0.1527(0.0216)$ & $0.8472(0.0216)$ \\
\hline
\end{tabular}

dictability performance for the Random Forest models.

This is supported by the results shown in Table 3 where the Random Forest models have $F_{1}$-scores around 0.75 , whereas the Random Guesser baseline have $F_{1}$-scores around than 0.15 . Further, the Random Forest model have mean AUC scores between 0.85 and 0.87 . In fact the $95 \%$ confidence interval of the AUC metric for each of the three class labels ChangeUser, Credit and Order were $0.87 \pm 0.028$, $0.85 \pm 0.011$ and $0.86 \pm 0.018$ respectively. Although the metrics are slightly lower compared to the results from the first experiment, this similarly indicates the potential in predicting TTR for e-mails.

Similar to Figure 4a, Figure 5a indicates that the model have a higher misclassification for label 0 and 5 than the other labels, indicating that a TTR within 2 hours and beyond 48 hours are more difficult to predict. Two things are different from Figure $4 \mathrm{a}$. First, label 3 is also more difficult to predict. Second, the misclassifications are slightly worse than for Figure 4a.

\section{ANALYSIS AND DISCUSSION}

The results presented in Section 5 indicates that it is possible to predict the response time for customer support agents, as well as the response time for when customers will respond to e-mails received. Organizations can benefit from these conclusions in (at least) two scenarios. First, by aggregating the customer TTR, it is possible to more accurately predict the workload of agents the next couple of days. This can be useful for either increasing the workforce, or shifting personnel working on other topics. Secondly, given that a predicted customer TTR is low, it might be advisable for support agents to focus on other emails with a low predicted agent TTR while waiting, in order to be able to respond quickly the customers eventual reply.

Predicting the support agents TTR is also useful since it can be used as a proxy to indicate the emotional and cognitive load associated with each email (Rafaeli et al., 2019), enabling more experienced agents to handle them, planning the agents' workload (e.g. several low agent TTR e-mails, or a few long agent TTR e-mails). Further, predicting agent TTR 
allow for customers to be alerted when a support errand is predicted to take a longer time than usual.

Even though the feature set of this experiment is based partly on related work and partly on domain expertise, it is important to investigate that the models have not learnt trivial solutions. For this reason, a random tree in the Random Forest model has been extracted and visualized using the Graphviz framework (Gansner and North, 2000), of which a sub-tree can be seen in Figure 6. This tree indicates that the model has indeed not learnt a trivial solution when predicting TTR.

As a way to further investigate the internals of the models trained by Random Forest, the ELI5 model interpretation framework was used to estimate the features' impact on the model's class assignment. Table 4 shows the relative impact each feature has on the predictive result in the Random Forest model. The most highly ranked feature is Sender followed by the Message length and Text complexity, which seem reasonable.

Table 4: Feature Weights for a Model Predicting Customer TTR.

\begin{aligned} & \hline Weight Feature \\ & \hline $0.2988 \pm 0.0899$ Sender \\ & $0.2094 \pm 0.0770$ Message length \\ & $0.2092 \pm 0.0772$ Text complexity factor \\ & $0.1685 \pm 0.0724$ Text sentiment \\ & $0.0598 \pm 0.0365$ Old $\square \\ & 0.0544 \pm 0.0322$ Customer escalated \\ & $0 \pm 0.0000$ Escalated \\ & \hline\end{aligned}

An example of an instance being predicted using a Random Forest model can be seen in Figure 7, where the probability and feature impact is shown for each possible response time bin. This particular instance, is a true positive as it is correctly assigned to the $4-8 \mathrm{~h}$ bin with an accuracy of $0.98 \%$. The most significant feature in favor for this decision is Sender that is assigned a weight of +0.324 . The feature BIAS is the expected average score based on the distribution of the data ${ }^{2}$. In this case, the BIAS is quite similar between the classes as the data, after the preprocessing, are balanced between the classes. Overall, this analysis of feature impacts indicate that the model has picked up patterns in the e-mails that are relevant for predicting TTR. Thus, it can be concluded that the models have not learned useless patterns from arte-

\footnotetext{
${ }^{2}$ https://stackoverflow.com/questions/49402701/eli5explaining-prediction-xgboost-model, accessed: 2020-0215
}

facts in the data sets.

The results from both experiments in this study suggests that the models' performance are in line with results from related research in the problem domain of instant messaging: 0.71 accuracy (Pielot et al., 2014), 0.89 accuracy (Huang and $\mathrm{Ku}, 2018$ ), 0.90 accuracy (Avrahami and Hudson, 2006). These results compare well to the results in this study. See Table 2 and Table 3 that have accuracy scores between $0.81-0.87$ and $0.74-0.77$ respectively. Thus, compared to the state-of-the-art, the results presented in this study indicates improved performance $(A U C \approx$ $0.85, F_{1} \approx 0.84$, accuracy $\approx 0.82$ in mean performance for agent TTR), compared to the best performing model among related work that was ADABoost $\left(A U C=0.72, F_{1}=0.45\right.$, accuracy $\left.=0.46\right)$ (Yang et al., 2017).

Finally, the problem was investigated separately for support agents and for customers. The results suggest that it is easier to predict the time to respond for agents, than it is for customers (c.f. Figure 4a and Figure 5a). This supports the prior statement that in this setting the agents can be considered a more homogeneous group due to similar training and experience, whereas the customers could be regarded as a more heterogeneous group of persons with different background and experiences.

\section{CONCLUSION AND FUTURE WORK}

This study investigated the ability to predict e-mail time-to-reply for both customer support agents as well as customers in a customer support setting. The results indicate that it is possible to predict the time agents will take to reply to an e-mail with an AUC of 0.90 , using seven features extracted from the e-mails. Further, that it is possible to predict the time-to-reply for customers to respond with an AUC of 0.85. These conclusions can be used to anticipate the staff needs in customer support, but also indicate to customers when an e-mail might take a longer time than expected to respond to. Additionally, given that timeto-reply can be indicative of emotional and cognitive load, it can also be used to better tailor message prioritization, e.g. messages with a high cognitive load might be more efficiently handled by senior customer agents, and messages with a lower cognitive load by junior customer agents.

As future work, it would be interesting to evaluate this prediction in practice. Such an evaluation would be two-fold. First, to what extent can this be used to more effectively predict workload of the customer 


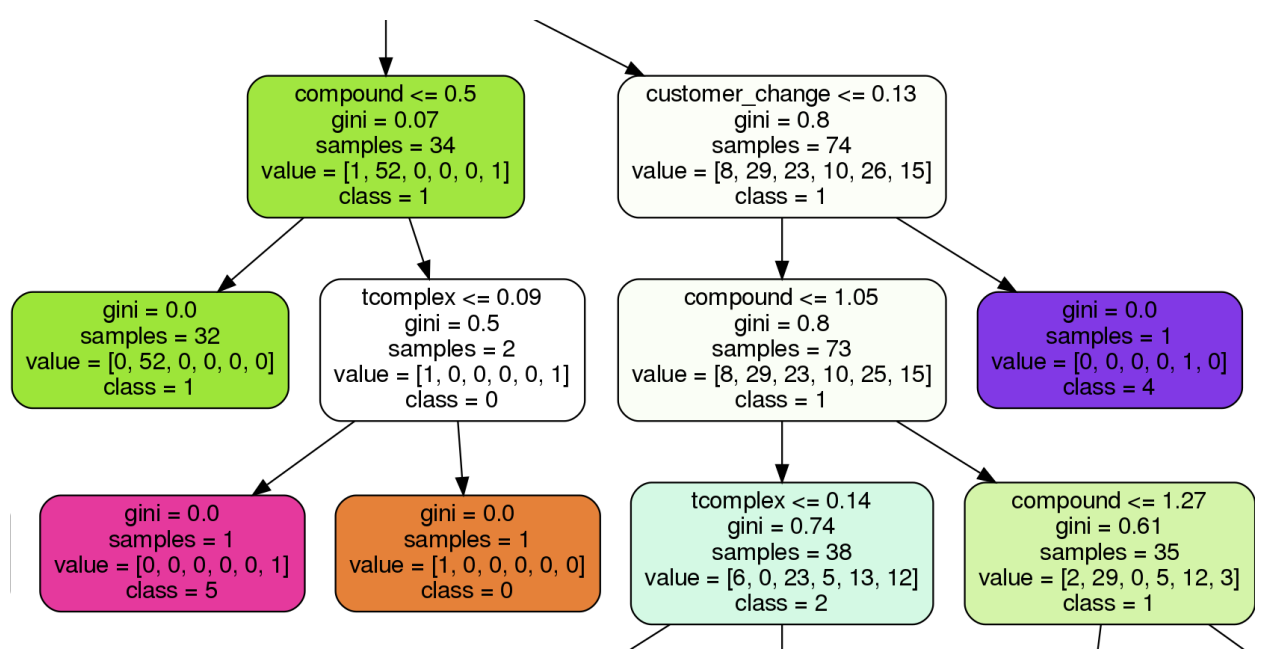

Figure 6: Sub-Tree Extracted from a Random Tree in a RF Model.

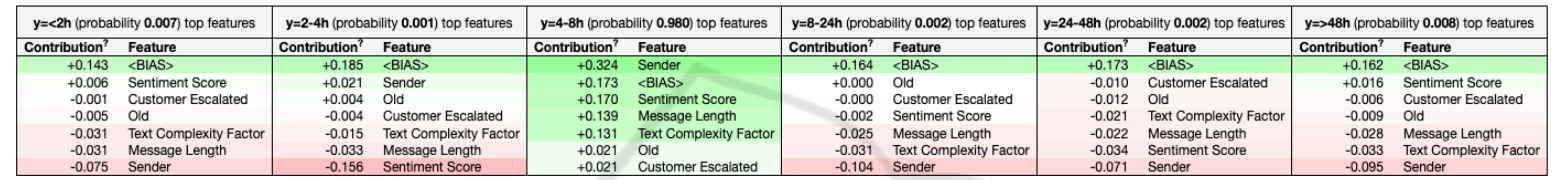

Figure 7: Prediction Explanation for a Random Instance in the Test Set for Customer TTR Prediction. The Instance Is a True Positive, Where an Response Were Sent between 4-8 Hours from Receiving the E-Mail.

agents. Second, does it actually improve efficiency to map cognitive and emotional load to different agents based on experience.

\section{REFERENCES}

Abdallah, E., Abdallah, A. E., Bsoul, M., Otoom, A., and Al Daoud, E. (2013). Simplified features for email authorship identification. International Journal of Security and Networks, 8:72-81.

Avrahami, D., Fussell, S. R., and Hudson, S. E. (2008). Im waiting: Timing and responsiveness in semisynchronous communication. In Proceedings of the 2008 ACM Conference on Computer Supported Cooperative Work, CSCW '08, pages 285-294, New York, NY, USA. ACM.

Avrahami, D. and Hudson, S. E. (2006). Responsiveness in instant messaging: Predictive models supporting inter-personal communication. In Proceedings of the SIGCHI Conference on Human Factors in Computing Systems, CHI '06, pages 731-740, New York, NY, USA. ACM.

Baron, N. S. (1998). Letters by phone or speech by other means: the linguistics of email. Language \& Cотmunication, $18(2): 133$ - 170.

Batista, G. E., Bazzan, A. L., and Monard, M. C. (2003). Balancing training data for automated annotation of keywords: a case study. In $W O B$, pages 10-18.

Breiman, L. (2001). Random forests. Machine Learning, 45(1):5-32.
Church, K. and de Oliveira, R. (2013). What's up with whatsapp?: Comparing mobile instant messaging behaviors with traditional sms. In Proceedings of the 15th International Conference on Human-computer Interaction with Mobile Devices and Services, MobileHCI '13, pages 352-361, New York, NY, USA. ACM.

Farr, J. N., Jenkins, J. J., and Paterson, D. G. (1951). Simplification of flesch reading ease formula. Journal of applied psychology, 35(5):333.

Fawcett, T. (2004). Roc graphs: Notes and practical considerations for researchers. Machine learning, 31(1):138.

Flach, P. (2012). Machine learning: the art and science of algorithms that make sense of data. Cambridge University Press.

Gansner, E. R. and North, S. C. (2000). An open graph visualization system and its applications to software engineering. SOFTWARE - PRACTICE AND EXPERIENCE, 30(11):1203-1233.

Halpin, N. (2016). The customer service report: Why great customer service matters even more in the age of ecommerce and the channels that perform best.

Huang, C. and Ku, L. (2018). Emotionpush: Emotion and response time prediction towards human-like chatbots. In 2018 IEEE Global Communications Conference (GLOBECOM), pages 206-212.

Hutto, C. and Gilbert, E. (2015). Vader: A parsimonious rule-based model for sentiment analysis of social media text.

Ikoro, G. O., Mondragon, R. J., and White, G. (2017). Pre- 
dicting response waiting time in a chat room. In 2017 Computing Conference, pages 127-130.

Kooti, F., Aiello, L. M., Grbovic, M., Lerman, K., and Mantrach, A. (2015). Evolution of conversations in the age of email overload. In Proceedings of the 24th International Conference on World Wide Web, WWW '15, pages 603-613, Republic and Canton of Geneva, Switzerland. International World Wide Web Conferences Steering Committee.

Lemaître, G., Nogueira, F., and Aridas, C. K. (2017). Imbalanced-learn: A python toolbox to tackle the curse of imbalanced datasets in machine learning. Journal of Machine Learning Research, 18(17):1-5.

Pedregosa, F., Varoquaux, G., Gramfort, A., Michel, V., Thirion, B., Grisel, O., Blondel, M., Prettenhofer, P., Weiss, R., Dubourg, V., Vanderplas, J., Passos, A., Cournapeau, D., Brucher, M., Perrot, M., and Duchesnay, E. (2011). Scikit-learn: Machine learning in Python. Journal of Machine Learning Research, 12:2825-2830.

Pielot, M., de Oliveira, R., Kwak, H., and Oliver, N. (2014). Didn't you see my message?: Predicting attentiveness to mobile instant messages. In Proceedings of the $32 N d$ Annual ACM Conference on Human Factors in Computing Systems, CHI '14, pages 3319-3328, New York, NY, USA. ACM.

Rafaeli, A., Altman, D., and Yom-Tov, G. (2019). Cognitive and emotional load influence response time of service agents: A large scale analysis of chat service conversations. In Proceedings of the 52nd Hawaii International Conference on System Sciences.

Tsoumakas, G., Katakis, I., and Vlahavas, I. (2010). Mining Multi-label Data, pages 667-685. Springer US, Boston, MA.

Yang, L., Dumais, S. T., Bennett, P. N., and Awadallah, A. H. (2017). Characterizing and predicting enterprise email reply behavior. In Proceedings of the 40th International ACM SIGIR Conference on Research and Development in Information Retrieval, SIGIR '17, pages 235-244, New York, NY, USA. ACM.

Yang, Y. (1999). An evaluation of statistical approaches to text categorization. Information Retrieval, 1(1):6990. 